\title{
The LHCb upgrade
}

\section{Tomasz Szumlak ${ }^{1}$}

AGH - University of Science and Technology

al. Mickiewicza 30, 30-059 Kraków, Poland

E-mail: szumlakeagh.edu.pl

The LHCb experiment is designed to perform high-precision measurements of CP violation and search for New Physics using the enormous flux of beauty and charmed hadrons produced at the LHC. The operation and the results obtained from the data collected in 2010 and 2011 demonstrate that the detector is robust and functioning very well. However, the limit of $1 \mathrm{fb}-1$ of data per year cannot be overcome without improving the detector. We therefore plan for an upgraded spectrometer by 2018 with a $40 \mathrm{MHz}$ readout and a much more flexible softwarebased triggering system that will increase the data rate as well as the efficiency specially in the hadronic channels. Here we present the LHCb detector upgrade plans, based on the recently submitted Letter of Intent

The XIth International Conference on Heavy Quarks and Leptons, Prague, Czech Republic

June 11-15, 2012

1 Speaker 


\section{Introduction}

The LHCb Collaboration decided that in order to expand the current physics programme of the experiment it is necessary to upgrade the spectrometer [1] (see Fig. 1) to $40 \mathrm{MHz}$ readout with a software based High Level Trigger (HLT). This is critical for increasing both the data rate and trigger efficiency which, in turn, will lead to substantially higher annual yields (compared to the presently obtained) by a factor of ten for the muonic channels and around twenty for decays with fully hadronic final states. Apart from a large increase in sensitivity for various current "golden" observables, e.g.: $\mathrm{B}_{\mathrm{d}, \mathrm{s},} \rightarrow \mu \mu$ branching fraction or forward-backward asymmetry - $A_{\mathrm{FB}}$ - measured in the $\mathrm{B}_{\mathrm{d}} \rightarrow \mathrm{K}^{*} \mu \mu$ channel, the upgraded detector will allow study of lepton sector, electroweak physics or long lived exotic states. In general one can say that we aim to change the present "high precision" experiment into a "discovery" one. In this scenario the upgraded $\mathrm{LHCb}$ spectrometer will act as an universal purpose detector in forward direction.

In the following chapter we will shortly introduce physics motivation for the upgrade and technical issues related to the LHC machine respectively. In section 3 we discuss in detail the $\mathrm{LHCb}$ detector modifications that are necessary to go to $40 \mathrm{MHz}$ readout. This part will be followed by the conclusions.

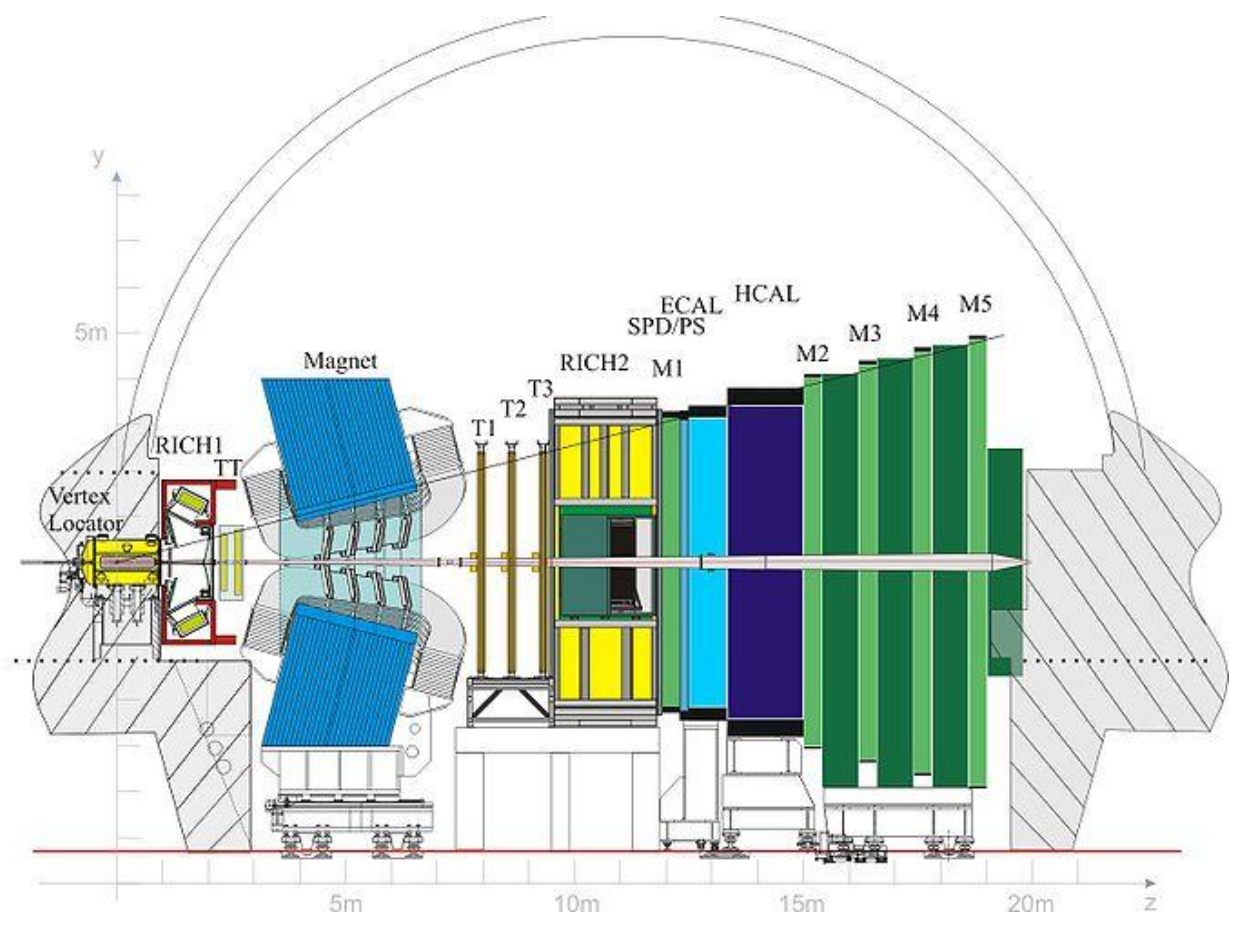

Figure 1: Cross-section of the LHCb detector in vertical plane. 


\section{Physics motivation}

The main goal of the upgrade project for the LHCb experiment is to excel in the field of flavour physics for both quark and lepton sectors and beyond. We would like to achieve this by enhancing the established strengths of the current design such as the forward acceptance, precise vertexing close to the interaction point and good particle identification capabilities. Also, we intend a major change of the High Level Trigger (HLT) that would allow it to operate at the 40 $\mathrm{MHz}$ rate in flexible way. All of these changes will allow the upgraded detector to run stably at the instantaneous luminosity up to $2 \times 10^{33} \mathrm{~cm}^{-2} \mathrm{~s}^{-1}$ and enable it to collect a large data sample of the order of $50 \mathrm{fb}^{-1}$.

In the following part of this chapter we will concentrate on the contribution of the upgraded LHCb experiment to the field of quark flavour physics. These predictions are based on the superb results obtained with first data. Is should be stressed that measurements in the hadronic flavour sector (regarding beauty and charm hadrons) have great sensitivity to New Physics phenomena. With the large data sample that can be accumulated after the upgrade we will be able not only to discover the new effects but also to characterise its nature when found. The large sample of events collected with the upgraded spectrometer will allow to perform a number of super-precise measurements of fundamental flavour quantities such the CKM angle $\gamma$. A short review of expected sensitivities for a number of the most important observables is given in Table 1 [2-3].

\begin{tabular}{|c|c|c|c|c|c|}
\hline Type & Observable & $\begin{array}{l}\text { Current } \\
\text { precision }\end{array}$ & $\begin{array}{c}\mathrm{LHCb} \\
\left(5 \mathrm{fb}^{-1}\right)\end{array}$ & $\begin{array}{l}\text { Upgrade } \\
\left(50 \mathrm{fb}^{-1}\right)\end{array}$ & $\begin{array}{c}\text { Theory } \\
\text { uncertainty }\end{array}$ \\
\hline \multirow{3}{*}{$\begin{array}{l}\text { Gluonic } \\
\text { penguin }\end{array}$} & $S\left(B_{s} \rightarrow \phi \phi\right)$ & - & 0.08 & 0.02 & 0.02 \\
\hline & $S\left(B_{s} \rightarrow K^{* 0} K^{* 0}\right)$ & - & 0.07 & 0.02 & $<0.02$ \\
\hline & $S\left(B^{0} \rightarrow \phi K_{S}^{0}\right)$ & 0.17 & 0.15 & 0.03 & 0.02 \\
\hline$B_{s}$ mixing & $2 \beta_{s}\left(B_{s} \rightarrow J / \psi \phi\right)$ & 0.35 & 0.019 & 0.006 & $\sim 0.003$ \\
\hline \multirow{2}{*}{$\begin{array}{l}\text { Right-handed } \\
\text { currents }\end{array}$} & $S\left(B_{s} \rightarrow \phi \gamma\right)$ & - & 0.07 & 0.02 & $<0.01$ \\
\hline & $\mathcal{A}^{\Delta \Gamma_{s}}\left(B_{s} \rightarrow \phi \gamma\right)$ & - & 0.14 & 0.03 & 0.02 \\
\hline \multirow{2}{*}{$\begin{array}{c}\mathrm{E} / \mathrm{W} \\
\text { penguin }\end{array}$} & $A_{T}^{(2)}\left(B^{0} \rightarrow K^{* 0} \mu^{+} \mu^{-}\right)$ & - & 0.14 & 0.04 & 0.05 \\
\hline & $s_{0} A_{\mathrm{FB}}\left(B^{0} \rightarrow K^{* 0} \mu^{+} \mu^{-}\right)$ & - & $4 \%$ & $1 \%$ & $7 \%$ \\
\hline \multirow{2}{*}{$\begin{array}{c}\text { Higgs } \\
\text { penguin }\end{array}$} & $\mathcal{B}\left(B_{s} \rightarrow \mu^{+} \mu^{-}\right)$ & - & $30 \%$ & $8 \%$ & $<10 \%$ \\
\hline & $\frac{\mathcal{B}\left(B^{0} \rightarrow \mu^{+} \mu^{-}\right)}{\mathcal{B}\left(B_{s} \rightarrow \mu^{+} \mu^{-}\right)}$ & - & - & $\sim 35 \%$ & $\sim 5 \%$ \\
\hline \multirow{3}{*}{$\begin{array}{c}\text { Unitarity } \\
\text { triangle } \\
\text { angles }\end{array}$} & $\gamma\left(B \rightarrow D^{(*)} K^{(*)}\right)$ & $\sim 20^{\circ}$ & $\sim 4^{\circ}$ & $0.9^{\circ}$ & negligible \\
\hline & $\gamma\left(B_{s} \rightarrow D_{s} K\right)$ & - & $\sim 7^{\circ}$ & $1.5^{\circ}$ & negligible \\
\hline & $\beta\left(B^{0} \rightarrow J / \psi K^{0}\right)$ & $1^{\circ}$ & $0.5^{\circ}$ & $0.2^{\circ}$ & negligible \\
\hline \multirow{2}{*}{$\begin{array}{l}\text { Charm } \\
\text { CPV }\end{array}$} & $A_{\Gamma}$ & $2.5 \times 10^{-3}$ & $2 \times 10^{-4}$ & $4 \times 10^{-5}$ & - \\
\hline & $A_{C P}^{d i r}(K K)-A_{C P}^{d i r}(\pi \pi)$ & $4.3 \times 10^{-3}$ & $4 \times 10^{-4}$ & $8 \times 10^{-5}$ & - \\
\hline
\end{tabular}

Table 1. Statistical sensitivities of the LHCb upgrade to key observables. For each observable the current sensitivity is compared to that expected after LHCb has accumulated $5 \mathrm{fb}^{-1}$ and 50 $\mathrm{fb}^{-1}$ respectively.

The scientific goals for the upgraded LHCb, however, extend far beyond quark-flavour sector. The unique acceptance of the spectrometer together with flexible software trigger will 
facilitate searches for long-lived exotic particles and detailed study of the weak mixing angle. A full review is given in paper [3].

\subsection{Impact of the LHCb upgrade on the LHC machine}

Although the target instantaneous luminosity for the upgrade of the LHCb experiment is much lower than the nominal LHC luminosity it has serious implications for its operation [4]. Because of the luminosity levelling procedure a virtual luminosity at the beginning of each fill must be close to the designed one for the machine, i.e., $\sim 1 \times 10^{34} \mathrm{~cm}^{-2} \mathrm{~s}^{-1}$. This in turn implicates that a special measures need to be taken to protect the machine against particles leaving the interaction point at this luminosity. Also, in order to maintain the luminosity at $2 \times 10^{33} \mathrm{~cm}^{-2} \mathrm{~s}^{-1}$ throughout the fill both beams must be focused to a $\beta^{*}$ about $3.5 \mathrm{~m}$. This has a significant consequences on the optical system of the LHC and requires implementation of the optimised Achromatic-Telescopic Squeezing scheme. The first simulations and tests are very encouraging. A different problem related to both physics data and the machine optics is the need to take the data at both polarisation of the LHCb dipole magnet. This is essential for the study of systematic effects that are important for precise asymmetry measurements.

Another issue of great importance is the protection against increased radiation. This will especially has influence on sensitive electronic control and monitoring equipment. Some of the PLC (programmable logic controllers) will be relocated during the first long shutdown of the machine.

\section{Modifications to the detector's sub-systems}

In order to cope with the larger luminosity, increased pile-up and $25 \mathrm{~ns}$ bunch spacing a number of the LHCb spectrometer's sub-systems need to be upgraded. Below we discuss briefly the plans for tracking and particle identification systems.

\subsection{Tracking}

The current tracking system of the LHCb experiment consists of the Vertex Locator (VELO) detector and TT (Trigger Tracker) both upstream to the magnet and the Tracking stations downstream to the magnet. The VELO and TT are the silicon strip based detectors whilst the $\mathrm{T}$ stations are made in hybrid technology. The inner part (Inner Tracker - IT) is silicon based while the outer part (Outer Tracker - OT) is made of gas straw tubes.

There are two options currently studied for the upgraded VELO. The first uses the modified planar sensors with larger number of channels (2560 comparing to the present 2048) readout by new ASIC (Application Specific Integrated Circuit) capable of on-chip digital processing and zero suppression, that is being designed by the Krakow group [5]. The second option is based on pixel sensors bump bonded to the VeloPix readout ASIC based on the latest MediPix3 chip [6].

There is currently one option for the upgraded TT detector based on micro strip $300 \mu \mathrm{m}$ thick silicon sensors. The new TT will feature finer segmentation and full coverage within the detector acceptance. The new ASIC from Krakow is at the moment regarding as the only option for the readout of the upgraded TT.

Detailed simulations showed that the expected occupancy at the high luminosity at the central region of the Tracking stations will be too high for the gas straw tube modules to cope. 
Thus the $\mathrm{T}$ stations need to be completely redesigned. There are two leading options at the moment: the first with large area Inner Tracker build with silicon micro strip sensors and silicon scintillating-fibres based modules replaces the whole central section of the $\mathrm{T}$ stations. In both designs a number of gas modules are still needed to cover the remaining acceptance. The new front-end electronics compatible with $40 \mathrm{MHz}$ readout would be based on the new ASIC chip.

\subsection{Particle identification}

The LHCb PID (Particle Identification) system comprises of upstream and downstream (with respect to the dipole magnet) Cherenkov detectors, calorimeters and muon chambers. The intended changes to these sub-detectors are described below.

The essential part of the current PID system of the LHCb experiment is two RICH (Ring Imaging Cherenkov) detectors or RICH-1 and RICH-2. The former will retain its current gas radiator $\mathrm{C}_{4} \mathrm{~F}_{10}$ but the aerogel radiator will be removed. The latter detector's radiator will remain unchanged. All of the HPD photon detectors and readout electronics will need to be replaced by mulit-anode photomulitpliers (MaPMTs) with external readout electronics capable of sending data at $40 \mathrm{MHz}$. All of the current mechanical and optical infrastructure will be reused as much as possible.

The current modernisation option for the calorimeter system is to replace completely the readout electronics. The new readout $40 \mathrm{MHz}$ ASIC must cope with significantly higher gain than the present one in order to compensate for a gain reduction that will be imposed on the photomultipliers. This imposes a tight requirement on the maximal noise level for the analogue part of the new chip. The rest of the calorimeters infrastructure will be kept apart form the Scintillating Pad Detector (SPD) and the Preshower (PRS) that most probably will be removed

\section{Conclusions}

The superb performance of the current LHCb spectrometer during 2011 and 2012 data taking are confirmed by the large number of important result (e.g., first evidence for CPviolation in $\mathrm{D}^{0}$ mesons decays). In order to expand the physics reach of the LHCb experiment and harvest the full potential of the LHC machine a challenging upgrade is being prepared. The most important features of this upgrade are fully software High Level Trigger capable of accessing the full event information at a frequency equal to the LHC beam crossing one. The total amount of data collected during the operation of the upgraded spectrometer is close to 50 $\mathrm{fb}^{-1}$. The upgrade plans has been initially endorsed by the CERN Council. The full Upgrade TDR will be published in 2013. 


\section{References}

[1] LHCb Collaboration, A. A. Alves et al., JINST 3 (2008) S08005

[2] LHCb Collaboration, I. Bediaga et al., Framework TDR for the LHCb upgrade, CERN-LHCC2012-007.

[3] LHCb Collaboration, R. Aaij et al., Letter of Intend for the LHCb Upgrade, CERN-LHCC-2011001

[4] B. Holzer et al., Optics and Lattice optimisations for the LHC upgrade project, Proceedings of IPAC 2012, New Orleans, May 2012

[5] Workshop on Common ASIC for the LHCb Upgrade (indico.cern.ch/conferenceDisplay.py?confId=194345)

[6] Medipix web site (medipix.web.cern.ch/MEDIPIX/) 